Journal of Neuroimmunology

Journal of Neuroimmunology 66 (1996) 125-134

\title{
Control of lymphocyte adhesion to brain and aortic endothelium: ICAM-1, VCAM-1 and negative charge
}

\author{
Washington L.C. dos Santos ${ }^{a}$, Jameel Rahman ${ }^{a}$, Nigel Klein ${ }^{b}$, David K. Male ${ }^{\text {a, * }}$ \\ a Department of Neuropathology, Institute of Psychiatry, De Crespigny Park, Denmark Hill, London SE5 8AF, UK \\ ${ }^{\mathrm{b}}$ Molecular Immunology Unit, Institute of Child Health, London, UK
}

Received 13 December 1995; accepted 15 February 1996

\begin{abstract}
Lymphocyte adhesion to CINS endothelium is low by comparison with non-CNS endothelium. It has been proposed that this could be due to the high surface charge of brain endothelium, or a low constitutive expression of adhesion molecules. In this study we compared the influence of these factors on lymphocyte adhesion to BEC and aortic endothelium (AEC) in culture. Brain endothelium expresses very low levels of VCAM-1, and lower levels of ICAM-1 than aortic endothelium. The negative charge differed between the endothelia, but this had a minimal effect on lymphocyte adhesion. The anionic sites were, however, more stable on brain endothelium, remaining unchanged after endothelial cell activation with cytokines, while redistribution was observed on cytokine-activated aortic endothelium.
\end{abstract}

Keywords: Endothelium; VCAM-I /VI A-4; ICAM-1/LFA1; Negative charge; I ymphocyte adhesion

\section{Introduction}

Lymphocyte traffic into the brain is usually very limited, although it increases in inflammatory conditions. The initial steps in lymphocyte migration include rolling on the endothelium, followed by adherence. Since adhesion precedes migration, it has been proposed that the low level of cell traffic through CNS is related to the low level of interaction between lymphocytes and brain endothelium. The levels of lymphocyte adhesion to brain endothelial cells (BECs) in vitro are also low, hy comparison with non-CNS endothelium even when it has been upregulated with TNF $\alpha$ or IFN $\gamma$ (Male et al., 1990; Pryce et al., 1991, 1994; May et al., 1993).

Among the actions performed by inflammatory cytokines, up-regulation of adhesion molecules seems to be the most important for leukocyte adhesion to the EC (Springer, 1994). A close relationship has been demonstrated between the up-regulation of selectins and immunoglobulin superfamily cell adhesion molecules (CAMs) on $\mathrm{EC}$ and increasing levels of leucocyte adhesion in vitro (Lowe et al., 1990; Shimizu et al., 1991; Male et al., 1994) and in vivo (Argenbright and Barton, 1992). Among the

\footnotetext{
${ }^{\star}$ Corresponding author. Tel. +44 (171) 9193286 ; Fax $+44(171) 708$ 3895 .
}

0165-5728/96/\$15.00 C 1996 Elsevier Science B.V. All rights reserved PII S0165-5728(96)00037-9 pathways involved in this interaction, that mediated by ICAM1/LFA1 is considered to be the more relevant for adhesion and migration (Shimizu et al., 1991; Kavanaugh et al., 1991; Oppenheimer-Marks et al., 1991). Another important pathway, at least in some leukocyte/EC interaction, is that involving VCAM1 /VLA4 (Carlos et al., 1991; Shimizu et al., 1991; Weller et al., 1991; Yednock et al., 1992). The importance of the interactions of ICAM1/LFA1 in the binding of lymphocytes to the brain endothelium has been well established (Male et al., 1994). Blocking studies, however, suggested the participation of other pathways in this interaction (Yednock et al., 1992).

Different populations of leukocytes use distinct sets of adhesion molecules to interact with endothelium, and the same is truc for EC. Differential expression of adhesion molecules during inflammation is suggested by studies in vitro and in vivo (Raine et al., 1990). Few studies, however have assessed the contribution of these systems to the lymphocyte adhesion and migration into different organs.

Besides the increase in adhesion molecules, another effect of cytokines on EC, potentially related to cell adhesion, is the induction of quantitative changes in glycosaminoglycans (GAG), observed on aortic endothelial cells (AEC, Kobayashi et al., 1990) and HUVEC (Klein et al., 1992), and in the distribution of the anionic sites observed on HUVECS (Klein et al., 1992). 
The anions on the EC surface are thought to be involved in regulating the transit of molecules and cells from the circulation, either exerting electrostatic repulsion (Bell et al., 1984) or by binding proteins, some of them relevant for leukocyte adhesion and migration (Tanaka et al., 1993a,b). Previous studies have also shown that negatively charged sites on EC are extremely sensitive to a number of biochemical and physiological stimuli (Klein et al., 1992; Kobayashi et al., 1990; Nag, 1984), and have been found to be altered in certain pathological conditions (Vorbrodt et al., 1990; Gotloib et al., 1992).

BEC differ from other EC in many respects. Compared with aortic or epididymal fat microcirculation EC, BEC show differences in the distribution of GAG-related anionic sites (Santos et al., 1995), and a lower ability to recruit lymphocytes. Therefore using BEC and AEC cultures, we addressed the question of the relative contribution of the negative charge and expression of adhesion molecules, to the levels of lymphocyte adhesion to different EC.

First we analyzed the effect of inflammatory cytokines (TNF $\alpha$ and IFN $\gamma$ ) on anionic sites on BEC and AEC. Then we analyzed the adhesion of lymphocytes to both endothelial cells under conditions interfering with the general surface charge, and with specific anions heparan sulfate (HS) and sialic acid. We also examined the expression of VCAM1 and ICAM1 on both resting or TNF $\alpha$-activated $\mathrm{EC}$, and correlated this with how these molecules are used by the different endothelia and lymphocytes.

\section{Material and methods}

\subsection{Endothelial cell monolayers}

Brain microvascular endothelia were isolated from 3 month-old, female Lewis rats (Hughes and Lantos, 1986). Cerebral cortex obtained by dissection, was chopped and digested for $1 \mathrm{~h}$ at $37^{\circ} \mathrm{C}$ in $15 \mathrm{ml} 0.1 \%(\mathrm{w} / \mathrm{v})$ collagenase/dispase (Boehringer), $10 \mu \mathrm{g} / \mathrm{ml}$ DNAse (Sigma), and $0.147 \mu \mathrm{g} / \mathrm{ml} N$-p-tosyl-L-lysine chloromethyl ketone (TLCK, Sigma) in $\mathrm{Ca}^{2+}$ - and $\mathrm{Mg}^{2+}$-free balanced salt solution (BSS, Gibco), containing $10 \mathrm{mM}$ HEPES, 100 $\mathrm{U} / \mathrm{ml}$ penicillin and $100 \mu \mathrm{g} / \mathrm{ml}$ streptomycin. Tissue was further disrupted by aspiration through a narrow-bore Pasteur pipette, and myelin removed by density fractionation in $250 \mathrm{mg} / \mathrm{ml} \mathrm{BSA}$ centrifuged at $1000 \times \mathrm{g}$ for $20 \mathrm{~min}$. The pellet was digested for a further $3 \mathrm{~h}$ at $37^{\circ} \mathrm{C}$, in $5 \mathrm{ml}$ of the collagenase/dispase solution, containing DNAse and TLCK (as above), resuspended in BSS, and separated from contaminating cells by centrifugation for $10 \mathrm{~min}$ on Percoll gradients, at $1000 \times \mathrm{g}$. (Gradients were established by centrifugation of $50 \%$ isotonic Percoll at $25000 \times g$ for 1 h.)

Aortic endothelia were obtained by placing small pieces
(2-3 $\left.\mathrm{mm}^{2}\right)$ of dissected rat aorta, luminal-side down on collagen-coated plates, in RPMI 1640 (Gibco) containing $10 \%$ fetal calf serum (FCS, Gibco), $75 \mu \mathrm{g} / \mathrm{ml}$ endothelial growth supplement (ECGS, Sigma), $40 \mu \mathrm{g} / \mathrm{ml}$ heparin, 2 $\mathrm{mM}$ glutamine, $100 \mathrm{U} / \mathrm{ml}$ penicillin and $100 \mu \mathrm{g} / \mathrm{ml}$ streptomycin $[\mathrm{P} / \mathrm{S}])$. After 3 days the explants were removed and the outgrowing endothelial cells expanded. They were passaged by trypsinization when confluent (McGuire and Orkin, 1987). Details of the characterization of brain and aortic endothelia prepared by these methods are presented elsewhere (Hughes and Lantos, 1986; Abbott et al., 1992; McGuire and Orkin, 1987).

BEC were grown in Ham's F-10 medium (Gibco) containing 20\% (v/v) bovine plasma-derived serum (PDS Advanced Protein Products) and AEC in RPMI- 1640 containing 20\% (FCS), both supplemented with $40 \mu \mathrm{g} / \mathrm{ml}$ heparin, $2 \mathrm{mM}$ glutamine, $\mathrm{P} / \mathrm{S}$, endothelial growth supplement (ECGS, Sigma). The cultures were maintained at $37^{\circ} \mathrm{C}$ in $5 \% \mathrm{CO}_{2}$, and fed every 2 days until they had formed confluent monolayers. Once confluent, the AEC were maintained in the same F-10 medium used for BEC. Although most of the characteristics of the aortic endothelium were preserved after passage, in these experiments only 1st passage endothelial cells were used.

\subsection{Lymphocytes}

Lymphocytes were obtained from peripheral and mesenteric lymph nodes of 8-14-week-old female Lewis rats, on the day before the adhesion assays. The lymph nodes were teased apart with forceps, into BSS and the cell suspension was passed through sterile gauze to remove clumps. The cells were washed three times in BSS before culturing overnight in RPMI-1640 containing 10\% FCS, $2 \mathrm{mM}$ glutamine, $\mathrm{P} / \mathrm{S}$. Activated cells were treated for $16-20 \mathrm{~h}$ before assay with $5 \mu \mathrm{g} / \mathrm{ml}$ concanavalin A (Con A, Sigma).

\subsection{Antibodies}

Anti-ICAM-1 (clone 1A29), anti-LFA-1(WT.1) and anti-CD18 (WT.3) are rat-specific antibodies generated in the laboratories of Dr. Tamatani and Dr. Miyasaka (Tokyo Metropolitan Institute of Medical Science). Anti-VLA-4 is a cross-reactive antibody raised to human VLA-4(clone HP2 / 1 from Immunotech, Marseilles, France). It is $\alpha$ chain-specific and inhibits VLA4 attachment to VCAM1 and fibronectin. Anti-VCAM1 (5F-10) supernatant was kindly donated by Dr. Ann Agar, from a clone generated by Dr Roy Lobb. It is a rat-specific antibody generated by fusion of spleen cells of mice immunized with CHO cells transfected with rat VCAM1. This antibody was shown to block the adhesion of lymphocytes to a recombinant human VCAM1 molecule (May et al., 1993). 


\subsection{Endothelial activation with cytokines}

The monolayers of BEC or AEC were washed with cold $\left(4^{\circ} \mathrm{C}\right) \mathrm{HBSS}$ and stimulated with either $100 \mathrm{U} / \mathrm{ml}$ of rat interferon- $\gamma$ (IFN $\gamma$, Holland Biotechnology) for $48 \mathrm{~h}$ or 25 $\mathrm{ng} / \mathrm{ml}$ recombinant human tumour necrosis factor- $\alpha$ (TNF $\alpha$, Genzyme) for $24 \mathrm{~h}$, in BECCM, without ECGS. In some experiments, higher doses of these cytokines were used, as detailed below.

\subsection{Lymphocyte adhesion assay}

The lymphocyte adhesion assay is based on that described previously (Male et al., 1990): resting or Con-Aactivated lymphocytes were washed twice in BSS and labelled with ${ }^{51} \mathrm{Cr}\left(3 \mu \mathrm{Ci} / 10^{6}\right.$ cells $)$ at $37^{\circ} \mathrm{C}$ for $90 \mathrm{~min}$. They were then washed and resuspended in BSS containing 10\% FCS. Labelled lymphocytes were applied to endothelial cell monolayers which had been washed four times with BSS. The wells for each treatment were randomized across the plates. The lymphocytes were cultured on the endothelium for $120 \mathrm{~min}$. Unbound cells were then removed by four washes in BSS at $37^{\circ} \mathrm{C}$. The bound and migrated cells were lysed in 2\% SDS and the lysate and one wash collected from each well. The number of bound/migrated cells was measured by counting released ${ }^{51} \mathrm{Cr}$. The results are expressed as percentage cells bound as a proportion of the number of cells applied $(=100 \%)$.

\subsubsection{Role of the negative charge on the endothelial cell surface on lymphocyte adhesion \\ The role of the negative surface charge on lymphocyte adhesion was examined, using threc differcnt systems:}

2.5.1.1. Lymphocyte adhesion to growing BEC. In a previous study we observed that the anionic sites related to heparin sulphate (HS) developed progressively in culture (Santos et al., 1995). For adhesion, therefore, we used BEC cultures on the 6th or 7th day, when only the central areas of the cell patches have the fully developed charge. The lymphocytes were allowed to adhere to BEC for 30 $\mathrm{min}$ and the cultures were immediately fixed with methanol or glutaraldehyde. Pictures were taken from different EC patches, and the cultures stained for negative charge with cationic colloidal gold (CCG), at $\mathrm{pH} 2.0$, as previously described (Klein et al., 1993). Briefly, EC cultures were fixed in absolute methanol at $-20^{\circ} \mathrm{C}$ for $15 \mathrm{~min}$, washed in BSS and stained with $5 \mathrm{~nm}$ cationic colloidal gold

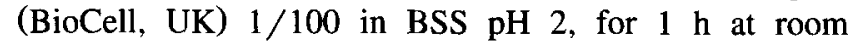
temperature. The reaction was developed with silver (Silver enhacer kit, Sigma), and counterstained with Harris' haematoxylin. The density of lymphocytes was calculated for the areas with low and high negative charge, and compared statistically. Whether the distribution of the lymphocytes was random was assessed by a Poisson test, and the differences between the density of lymphocytes attached to low and high charged areas of culture tested using Student's $t$-test.

\subsubsection{Interfering with endothelial cell negative charge} during lymphocyte adhesion. Brain or aortic endothelium were preincubated with $1 \mathrm{mg} / \mathrm{ml}$ lysine for $1-4 \mathrm{~h}$ at $37^{\circ} \mathrm{C}$ in F-10 cell media lacking heparin. The cells were washed as above, and overlaid with lymphocytes in BSS containing $10 \%$ FCS.

2.5.1.3. Enzyme treatment. Many of the anionic sites on BECs in culture are sensitive to neuraminidase and to heparitinase (Santos et al., 1995). HS is also an important anion on aortic endothelium in culture (Kobayashi et al., 1990). To investigate the impact of these anions on lymphocyte adhesion to BEC and AEC in culture, we selectively cleared the cell surface of sialic acid or HS: confluent monolayers were washed with BSS, and incubated with either $10 \mathrm{U} / \mathrm{ml}$ heparinase III (EC4.2.2.8), or $1 \mathrm{U} / \mathrm{ml}$ of neuraminidase type X (EC3.2.1.18, both from Sigma) in F-10 medium without heparin, for $4 \mathrm{~h}$ at $37^{\circ} \mathrm{C}$. After enzyme digestion, the cells were washed in BSS and maintained on ice, before co-incubation with lymphocytes. No morphological sign of endothelial cell damage was observed at the end of each incubation. In some experiments, the EC were fixed with formalin before enzyme digestion (Cabañas and Hogg, 1993). The effect of the enzymes on the anionic sites was checked in some experiments, by fixing with methanol or glutaraldehyde and staining with $\mathrm{CCG}$, at $\mathrm{pH} 2.0$, as described above.

\subsubsection{Expression of adhesion molecules on BEC and AEC}

Surface expression of ICAM1 and VCAM1, and MHC class I molecules on brain and aortic endothelium were measured by cell surface ELISA, using $3 \mu \mathrm{g} / \mathrm{ml} 1 \mathrm{~A} 29,1$ $\mu \mathrm{g} / \mathrm{ml} 5 \mathrm{~F}-10$, or $1 / 10$ OX18, respectively, followed by $1 / 700$ biotinylated anti-mouse Ig and 1/700 streptavidin peroxidase, developed with tetramethyl benzidine. The method has been described previously (Male et al., 1987). As a negative control, the first antibody was omitted or normal Ms IgG was used. As an additional control of the reaction on ELISA between experiments, empty wells of each plate were sensitized with sequential double dilutions of mouse IgG, from 2000 to $1.5 \mathrm{ng} / \mathrm{ml}$, and incubated with anti-mouse immunoglobulin and streptavidinperoxidase, as described above.

\subsubsection{Role of LFA-1 and $\alpha 4$-integrins on lymphocyte adhesion to AEC}

In the antibody-blocking experiments, ${ }^{51} \mathrm{Cr}$-labelled lymphocytes were preincubated for $10 \mathrm{~min}$ with anti- $\alpha 4$ or anti LFA-1, and then incubated with the endothelial cells. In order to see if FN was involved in the lymphocyte binding to the aortic or brain endothelium we used a fragment of FN CS-1 peptide (G.P.E.I.L.D.V.P.S.T), containing the L.D.V segment, shown to block the interaction of VLA-4 with fibronectin. This peptide was used at the 
concentrations of $10,50,100,250,500 \mu \mathrm{g} / \mathrm{ml}$, and as control, equimolar concentrations of a control peptide V.Y.W.T.P.E.R.V, were used.

\subsection{Significance of the results}

The EIA and the lymphocyte adhesion assays were performed in triplicate or quadruplicate, using wells randomized across the plate. The experiments with staining were performed in triplicate. The results presented here are representative of experiments performed on at least two different occasions. They are expressed as mean \pm S.E. of the O.D. $(450 \mathrm{~nm})$ for each antibody, relative to the expression of MHC class I molecule (OX18 antibody) or percentile of adherent lymphocytes, relative to the total added. The statistical significance of the differences between groups was tested using Student's $t$-test or one-way ANOVA, with critical level of significance at $P<0.05$.

\section{Results}

\subsection{Activation with inflammatory cytokines changes the distribution of anionic sites on AEC but not on BEC}

TNF $\alpha(25-150 \mathrm{ng} / \mathrm{ml})$ or IFN $\gamma(50-250 \mathrm{ng} / \mathrm{ml})$ in concentrations that up-regulate adhesion molecules and increase lymphocyte adhesion to brain endothelium (Male et al., 1990), failed to produce changes in intensity or distribution of the anionic sites on BEC. AEC cultured under the same conditions presented a more fibrillar distribution of the anionic sites when treated with TNF $\alpha$. Treatment with IFN $\gamma$ induced discrete aggregates of negatively charged material irregularly distributed among the cells. The changes were more intense when the cells were fixed with methanol. Although the redistribution of anions, following activation was evident on AEC grown in F-10 medium, its intensity contrasted with the profound changes
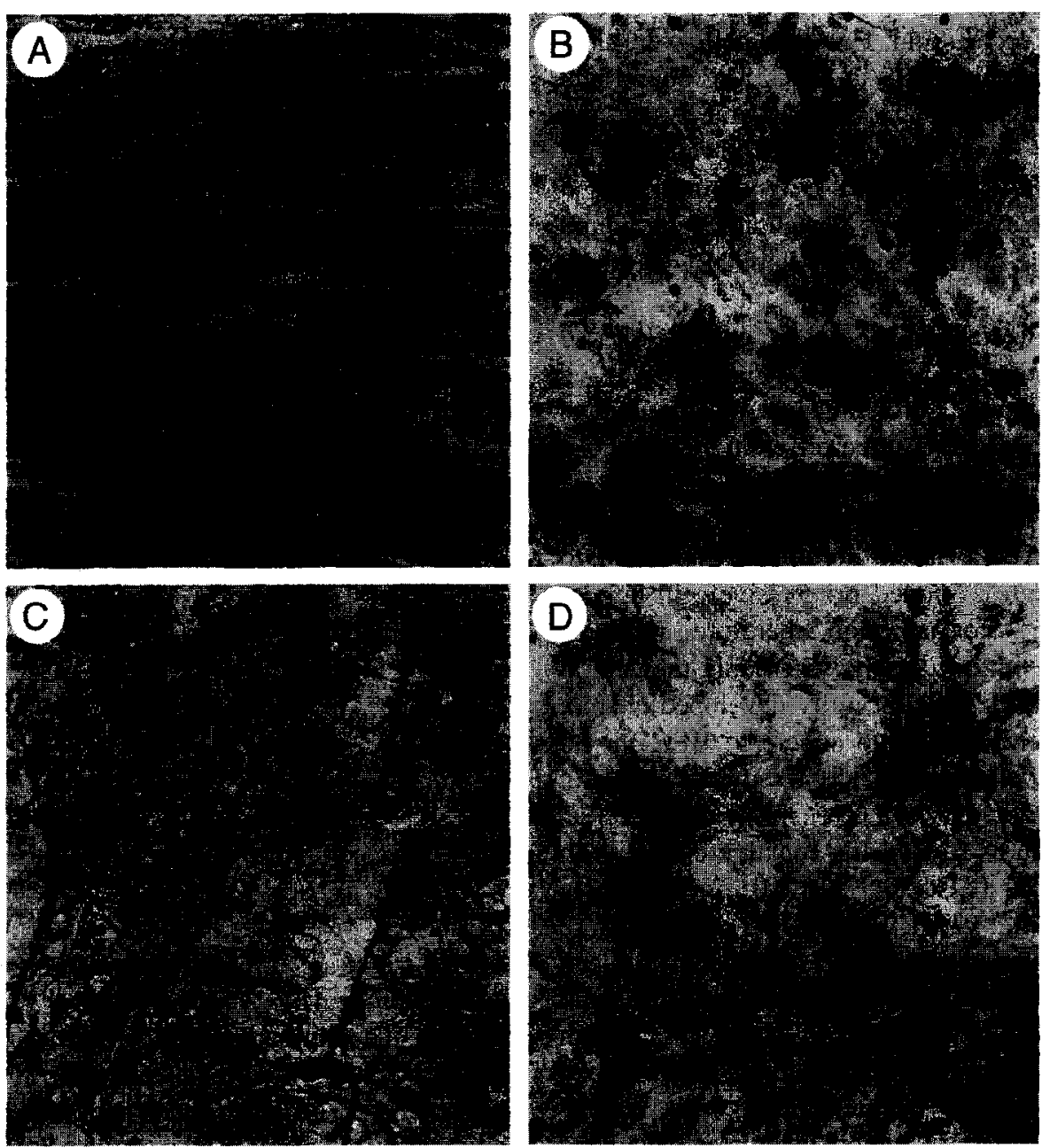

Fig. 1. TNF $\alpha(50 \mathrm{ng} / \mathrm{ml}, 24 \mathrm{~h})$ or IFN $\gamma(100 \mathrm{U} / \mathrm{ml}, 48 \mathrm{~h})$ change the distribution of the anionic sites on aortic (AEC) but not on brain (BEC) endothelial cells in culture. A. BEC: the negative charge is normally distributed at the periphery of the cells $(A)$, and remains unchanged after cytokine activation. B-D. AEC: on resting AEC the anionic sites distribute as a fine array of CCG particles all over the plasmalemma, with occasional fibrils at the periphery of the cells, and higher density in some areas (B). TNF $\alpha$ increases the intensity of the staining, and accentuates the fibrillar aspect (C). IFN $\gamma$ produces a complete redistribution of the anionic sites to form a network of fibrils (D). 
on GAGs, both biochemical and morphological reported on EC cultured in RPMI medium containing $20 \%$ FCS (Kobayashi et al., 1990; Klein et al., 1992). In order to verify if the conditions normally used for culture of BEC could account for the lack of change observed in our study, we performed some experiments in which the culture medium was replaced by RPMI containing $20 \%$ FCS and supplements, $48-96 \mathrm{~h}$ before fixation with methanol and staining with CCG. The BEC grown for $96 \mathrm{~h}$ in RPMI had a similar morphology and distribution of the anionic sites, to that seen with F-10 medium (Fig. 1A). This remained unchanged after activation with TNF $\alpha$ or IFN $\gamma$ (not shown). On AEC, changing to RPMI medium caused the anionic sites to distribute more uniformly. A fine array of CCG particles is observed all over the plasmalemma, with occasional fibrils at the periphery of the cells (Fig. 1B). TNF $\alpha$ increases the intensity of the staining, and accentuates the fibrillar aspect, producing aggregates and bundles of fibrils spanning many cells (Fig. 1C). Activation with IFN $\gamma$ produced a complete redistribution of the anionic sites to a network of fibrils (Fig. ID). After activation, the AEC changed in shape. With TNF $\alpha$ the culture became more pleomorphic, with sorne cells presenting a fusiform aspect. With IFN $\gamma$ some cells appeared contracted, producing areas of discontinuity on the monolayers.

\subsection{Lymphocyte adhesion to BEC in culture is indepen- dent of the distribution of $H S$}

The analysis of the distribution of the lymphocytes bound to the growing brain endothelium, using a Poisson test, showed that the binding of lymphocytes to the BEC in culture is non-random, but it is not influenced by the density of anionic sites related to HS (Fig. 2A and B). The difference in density of lymphocytes attached to areas of high or low negative anionic sites was never statistically significant. However the technique coincidentally demonstrated a high density of anionic sites at the leading edges of the leukocytes, where they contact the endothelial cell surface (Fig. 2C).

\subsection{Comparison of lymphocyte binding to BEC and AEC under conditions interfering with the negative surface charge}

The levels of lymphocyte adhesion to aortic endothelium were usually higher than to the brain endothelium. On the experiments presented here, the levels of adhesion of activated lymphocytes to AEC varied normally between 32 and $50 \%$ on resting $\mathrm{EC}$, increasing to $50-60 \%$ when the endothelial cells were activated with TNF $\alpha$. The levels of adhesion to resting BEC, were usually $14-21 \%$, increasing to $30 \%$ on activated cells. No significant change in adhesion occurred when the EC (AEC or BEC) were pretreated with L-lysine, or when L-lysine was maintained in the medium during the adhesion (Fig. 3).
The enzymes produced only minimal changes on lymphocyte adhesion to brain endothelium (Fig. 3). The levels of adhesion to aortic endothelium were usually lower after treatment with neuraminidase or heparitinase (Fig. 3). These changes were significant in only one of three experiments with neuraminidase and two of three experiments with heparitinase. On BEC, heparitinase reduced significantly the binding of lymphocytes in one experiment only (Fig. 3).

3.4. VCAM1 is virtually absent from resting BEC, and induced only to low levels after $T N F \alpha$ activation

Resting brain endothelium in culture expresses low levels of ICAMI and virtually undetectable levels of VCAM1. Both the molecules increase progressively on BEC. ICAM1 from $5(P<0.01)$ to $24 \mathrm{~h}(P<0.001)$, and VCAM1 from $12 \mathrm{~h}(P<0.001)$. VCAM1 starts to decline at $48 \mathrm{~h}$. TNF $\alpha$ was more effective in inducing the expression of VCAM1 in BEC than IFNy (not shown). In one experiment the time course of the expression of these molecules after activation with TNF $\alpha$, showed that, VCAMl expression returned to basal levels after $96 \mathrm{~h}$ on BEC. On AEC, ICAM1 and VCAM1 are constitutively expressed at high levels in culture, and increase slightly after $24-48 \mathrm{~h}$ of activation. The increase in expression of VCAMI on AEC was always statistically significant, from 12 to $24 \mathrm{~h}(P<0.001)$. The increase in the expression of ICAMl was always observed, but did not reach statistical significance in some experiments. Unlike BEC, the levels of both ICAM1 and VCAM1 remain on plateau on AEC during the $96 \mathrm{~h}$ of experiment (Fig. 4A and B).

\subsection{Increased lymphocyte adhesion to AEC corresponds to the induction of ICAMI and VCAMI}

Since we observed differences in the levels of expression of VCAM1 between brain and aortic endothelium, we decided to cxamine if such differences were also related to the use of pathways involving the ligands for VCAM1, through adhesion blocking expcriments, using an anti- $\alpha 4$ integrin antibody. This antibody was used during lymphocyte adhesion to BEC, and only produced consistent levels of blocking when used together with an anti-LFAl antibody (Male et al., 1994).

The levels of adhesion of resting lymphocytes to AEC were around $16 \%$. For activated lymphocytes the levels of adhesion to AEC varied normally between 32 and $51 \%$, and increased up to $61 \%$ when the endothelial cells were activated with TNF $\alpha$. As demonstrated for BEC (Male et al., 1990, 1994), the levels of lymphocyte binding to aortic endothelium also increased with EC activation, corresponding to the induction of ICAM1 and VCAM1 (Fig. 5A). The increased binding was observed from $5 \mathrm{~h}$ ( $P<$ $0.05)$ to $24 \mathrm{~h}(P<0.001)$.

Both antibodies against LFA1 and $\alpha 4$ integrins de- 

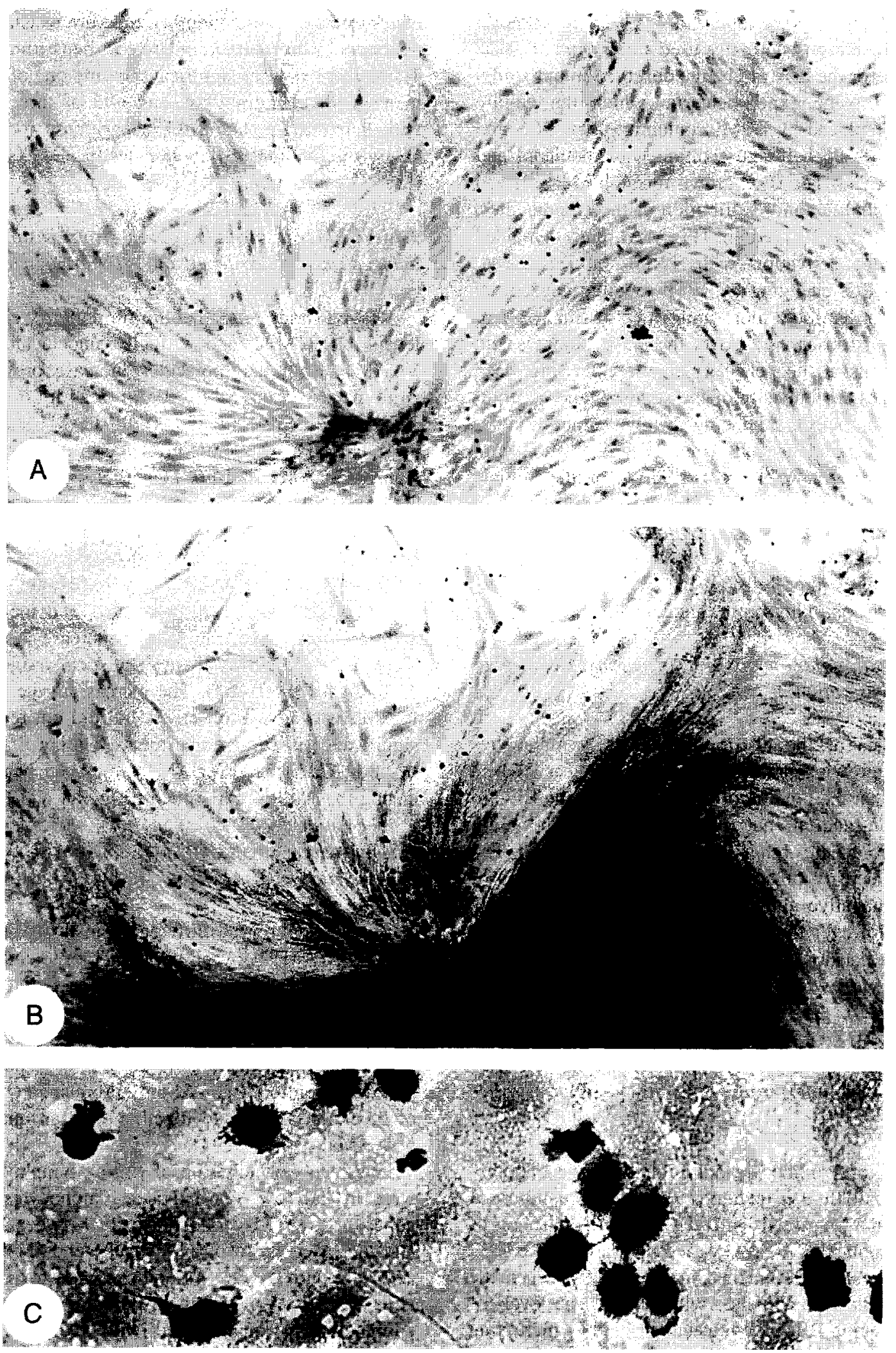

Fig. 2. A and B. Lymphocyte adhesion to non-confluent (5 d culture) BEC monolayer, methanol fixation and (A) haematoxylin staining, (B) cationic gold staining at $\mathrm{pH}$ 2.0. Anionic sites develop on the central areas of the cell patches. Lymphocytes adhere to brain endothelium independently of the intensity of charge on the cell surface. C. During the adhesion the anionic sites on the lymphocytes are redistributed to the areas of contact with the endothelium (glutaraldehyde fixation, CCG staining; $\mathrm{pH} 2.0$ ). 


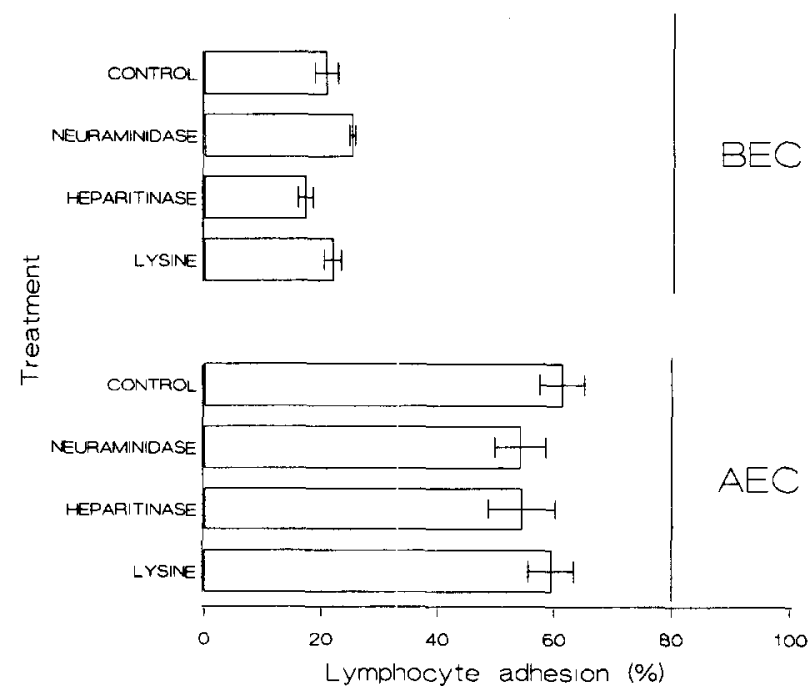

Fig. 3. Interfering with the negative charge on BEC or AEC, by preincubation with L-lysine $(1 \mathrm{mg} / \mathrm{ml})$ or pretreatment with neuraminidase type $\mathrm{X}(1 \mathrm{unit} / \mathrm{ml})$, or heparinase III (heparitinase, 5 units $/ \mathrm{ml}$ ), produces only small changes in the levels of lymphocyte adhesion.

creased the binding of lymphocytes to both activated or non-activated aortic endothelium (Fig. 5A and B). The levels of inhibition obtained with the anti- $\alpha 4$ antibody were $23-25 \%$ to non-activated AEC $(P<0.005)$, and $15 \%$ to activated endothelium $(P<0.01)$. The anti-LFA1 antibody was more effective on activated AEC, producing a $15-32 \%(P<0.05)$ decrease in lymphocyte binding, and only a $9-12 \%$ on non-activated endothelial cells. The two antibodies acted synergistically producing further reduction (up to $44 \%, P<0.005$ ) in lymphocyte adhesion. The fibronectin peptide used (CS-1) in concentrations as high as $500 \mu \mathrm{g} / \mathrm{ml}$ did not interfere with the adhesion of
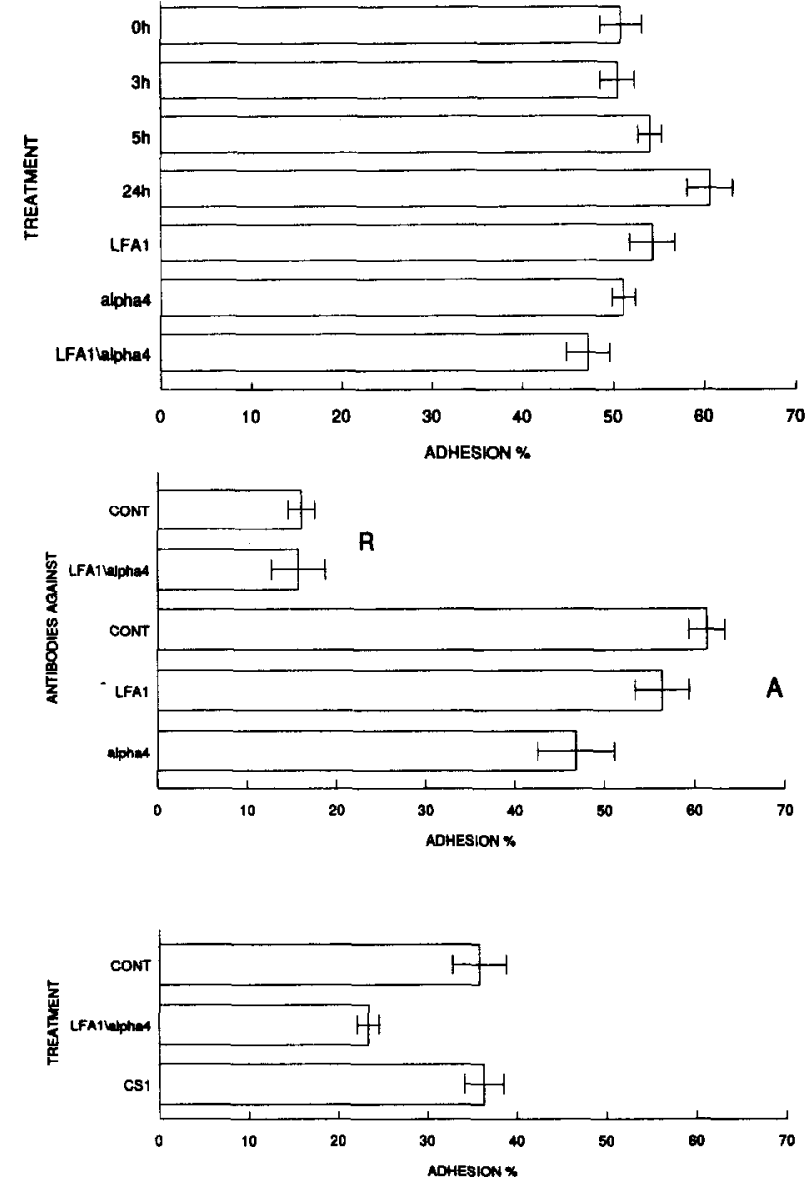

Fig. 5. Lymphocyte adhesion to AEC. Top: Adhesion of Con A-activated lymphocytes to AEC increases after treatment of the EC with TNF $\alpha$ (5-24 h). Monoclonal antibodies anti-LFA1 (WT1, $5 \mu \mathrm{g} / \mathrm{ml}$ ) or anti- $\alpha 4$ integrin ( $\mathrm{HP} 2 / 1,10 \mu \mathrm{g} / \mathrm{ml}$ ) inhibit the binding of activated lymphocytes to activated (top) or resting (centre) AEC, but do not change the adhesion of resting lymphocytes (centre: $R=$ resting, $A=$ activated lymphocytes). Bottom: No change was observed with the CS1 fragment of fibronectin.
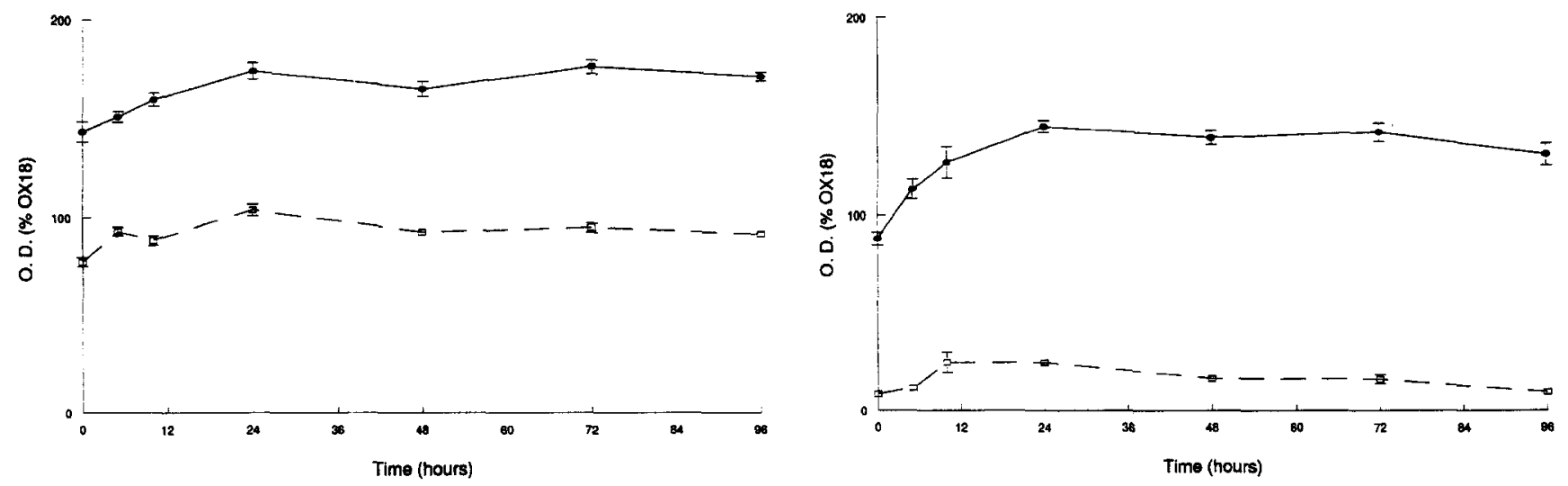

Fig. 4. ICAM1 (mAb 1A29, solid lines) and VCAM1 (mAb 5F10, broken lines) expression was measured on aortic (left) and brain (right) endothelium by cell surface ELISA. The monolayers were pulsed for $2-96 \mathrm{~h}$ with $25 \mathrm{ng} / \mathrm{ml} \mathrm{rTNF} \alpha$, before assay. Both the molecules increase progressively on BEC from 5 to $24 \mathrm{~h}$. ICAMl and VCAMl are constitutively expressed in high levels on cultured AEC and only increase slightly after 24 and $48 \mathrm{~h}$ of activation. The levels of ICAM1 are maintained in both AEC and BEC. The slight increase observed in VCAM1 expression on BEC subsides after $96 \mathrm{~h}$ of activation. 
activated lymphocytes to the AEC (Fig. 5C). Unlike the results obtained with activated lymphocytes, the anti- $\alpha 4$ antibody used alone, or together with anti-LFAl, antibody did not change the levels of adhesion of non-activated lymphocytes to AEC.

\section{Discussion}

The data presented here show that the anionic sites on AEC and BEC are regulated differently by inflammatory cytokines. While the anionic sites remain stable on BEC, they redistribute extensively on AEC stimulated with TNF $\alpha$ or IFN $\gamma$.

Redistribution or loss of EC surface anions has been described in a number of pathological conditions, affecting the brain (Nag, 1984; Vorbrodt et al., 1990) and other organs (Gotloib et al., 1992). The few studies using EC in vitro, show that the changes in the negative charge may occur as an early event in disease. It can be induced by the cytokines (Kobayashi et al., 1990; Klein et al., 1992) produced early at sites of inflammation, or result from direct damage by aluminium compounds (Vorbrodt and Trowbridge, 1993).

The EC surface anions have long been associated with the selective role of the EC in the transport of molecules and cells between blood and tissue (Hart et al., 1987; Mayhan et al., 1989). Our data shows that, unlike other $\mathrm{EC}$, the negative charge on $\mathrm{BEC}$ remains unchanged after activation by inflammatory cytokines.

Sincc our experiments werc carried out in non-flow conditions, they show that the negative surface charge is not a critical determinant of lymphocyte adhesion, once the contact has occurred between lymphocyte and BEC or AEC. Neither the distinct distribution, nor regulation of the surface anions on BEC and AEC, explain the differences in levels of lymphocyte adhesion to these EC.

Another interesting finding presented here, is the redistribution of molecules containing highly anionic sites (pI below 2.0) to the edges of the lymphocytes, during the adhesion to the BEC. Iossinsky et al. (1989) reported a similar finding in EAE. The redistribution of highly negatively charged molecules to the areas of cell-cell contact suggests their involvement in lymphocyte adhesion and/or migration. Further studies are, however, necessary for the characterization of these glycomers.

The expression of VCAM 1 is also distinct on BEC and AEC. On aortic endothelium the expression of VCAM1 follows a pattern similar to that described for high endothelial cells and HUVEC, in culture (May et al., 1993; Shimizu et al., 1991). However VCAMl is virtually absent from resting $\mathrm{BEC}$ and increases only to low levels after activation with TNF $\alpha$. This equates with the recent observations on the pathways involved in lymphocyte adhesion to and migration through BEC (Male et al., 1994. Pryce unpublished). VCAM1 was also found to be absent from acute lesions in MS (Raine, 1994) and was induced less effectively by LPS or TNF $\alpha$ than on meningeal endothelium, in vivo (Gotsch et al., 1994). On the other hand, increased expression of VCAM1 in the chronic phase of MS has been reported (Raine, 1994) and this molecule is apparently involved in the genesis of EAE (Yednock et al., 1992). This finding, together with the data presented here, suggest that the expression of VCAM1 and alterations on the negative charge, seen on BEC in some diseases, depends on additional or different stimuli from those needed to produce changes on AEC or HEV (May et al., 1993; Schmidley and Wissig, 1986).

On AEC, the increase of both ICAM1 and VCAM1 expression, following different periods of activation with TNF $\alpha$, corresponds to the time course of enhanced lymphocyte adhesion. Further evidence for the involvement of these molecules in this interaction, is given by the significant reduction in the binding produced by antibodies against LFAI or $\alpha 4$. Moreover, the anti- $\alpha 4$ antibody was more effective in blocking the binding of lymphocytes to both resting or activated AEC, than the anti-LFAI antibody.

Using anti- $\alpha 4$ antibodies, interactions mediated by the whole range of ligands for VLA4 or $\alpha 4 \beta 7$ integrins may be affected. In our experiments, however, the anti- $\alpha 4$ antibody had no effect on reducing the adhesion of resting lymphocytes to AEC, suggesting that $\alpha 4 \beta 7$ integrin/ MadCAM1 binding is not involved in this interaction. Apart from VCAM1, FN and thrombospondin (TSP) are ligands for VLA4 (Guan and Hynes, 1990; Yabkowitz et al., 1993). FN is potentially involved in the adhesion of leucocyte to some endothelial cells (Carlos and Harlan, 1994). Recently, sequences were identified in the 1 st and 4th domain of the VCAM1 molccule (Osborn et al., 1994; Clements et al., 1994) considered to play a critical role in the interaction with VLA-4. The homology shared between these segments and the LDV sequence on the CS-1 fragment of fibronectin, suggested that the sites used by VLA4 in the interaction with VCAMI and fibronectin, are overlapping or at least contiguous (Clements et al., 1994). In fact, cross-inhibition of cell attachment to fibronectin or recombinant VCAM1, has been demonstrated, using synthetic peptides from CS1 (May et al., 1993) and VCAM1 (Clements et al., 1994), respectively. In our experiments, however, we were not able to show a decrease in the adhesion of lymphocytes to the AEC, using the peptide from fibronectin. This peptide and related LDV-containing peptides have been shown to inhibit the binding of melanoma cells to fibronectin, even when used in molar concentrations one-tenth of that used here (Komoria et al., 1991). Elices et al. (1990) were not able to inhibit the binding of VLA4 transfected Ramos cells to HUVEC or VCAM1 transfected COS cells, using FN40. One possible explanation for the absence of blocking observed with the FN-peptide is the dynamic of the interactions of the integrins with their different receptors on the EC surface (Hogg et al,, 1993). Nevertheless, the absence of blocking 
of lymphocyte adhesion to AEC with the CS1 peptide, suggests that FN is not the ligand for VLA4 on aortic endothelium.

TSP is another potential source of differences in lymphocyte adherence to BEC and other EC. This molecule was only marginally up-regulated on BEC during cerebral malaria (Turner et al., 1994). Although the secretion of this molecule by AEC sharply decreases in confluent cultures (Mumby et al., 1984), further studies are necessary to establish any contribution to the high level of lymphocyte adhesion to aortic endothelium.

The residual levels of adhesion of activated lymphocyte to AEC, after blocking LFA1 and VLA4, is significantly higher than that observed for BEC, suggesting the differential participation of other molecules in the lymphocyte interaction with these cells.

In conclusion, our data shows that the negative charge on AEC is more responsive to activation with $\mathrm{TNF} \alpha$ and IFN $\gamma$ than the negative charge on BEC. VCAM1 is virtually absent from resting $B E C$, and increases to only low levels with EC activation. On the aortic endothelium high levels of VCAM1 and ICAM1 are expressed, and unlike our previous findings with BEC (Male et al., 1994), LFAl and $\alpha 4$ integrins are both used in lymphocyte interaction with AEC. The differences observed here can explain, at least in part, the usually low levels of lymphocyte adhesion to the BEC.

\section{Acknowledgements}

We are most grateful to Dr. Roy Lobb who made the anti-VCAM-1 antibody available to us. Washington Santos was supported by CNPQ (Brasil) and Jameel Rahman was supported by a grant from the Multiple Sclerosis Society of Great Britain.

\section{References}

Abbott, N.J., Hughes, C.C.W., Revest, P.A. and Greenwood, J. (1992) Development and characterization of rat brain capillary endothelial culture: towards an in vitro blood-brain barrier. J. Cell Sci. 103, 23-37.

Argenbright, L.W. and Barton, R.W. (1992) Interactions of leukocyte integrins with intercellular adhesion molecule 1 in the production of inflammatory vascular injury in vivo. The Shwartzman reaction revisited. J. Clin. Invest. 89, 259-272.

Bell, G.I., Dembo, M. and Bongrand, P. (1984) Cell adhesion competition between non specific repulsion and specific binding. Biophys. J. 45, 1051-1064.

Cabañas, C. and Hogg, N. (1993) Ligand intercellular adhesion molecule 1 has a necessary role in activation of integrin lymphocyte functionassociated molecule 1. Proc. Natl. Acad. Sci. USA 90, 5838-5842.

Carlos, T.M. and Harlan, J.M. (1994) Leukocyte-endothelial adhesion molecules. Blood 84, 2068-2101.

Carlos, T., Kovach, N., Schwartz, B., Rosa, M., Nawman, B., Wayner, E., Benjamin, C., Osborn, L., Lobb, R. and Harlan, J. (1991) Human monocytes bind to two cytokine-induced adhesive ligands on cultured human endothelial cells: endothelial-leukocyte adhesion molecule-1 and vascular cell adhesion molecule-1. Blood 77, 2266-2271.

Clements, J.M., Newham, P., Shepherd, M., Gilbert, R., Dudgeon, T.J., Needham, L.A., Edwards, R.M., Berry, L., Brass, A. and Humphries, M.J. (1994) Identification of a key integrin-binding sequence in VCAM-1 homologous to LDV active site in fibronectin. J. Cell Sci. $107,2127-2135$.

Elices, M.J., Osborn, L., Takada, Y., Crouse, C., Luhowsky, J.S., Hamler, M.E. and Lobb, R. (1990) VCAM-1 on activated endothelium interacts with the leukocyte intcgrin VLA-4 at sitc distinct from the VLA-4/fibronectin binding site. Cell 60, 577-584.

Gotloib, L., Shostak, A., Galdi, P., Jaichenko, J. and Fudin, R. (1992) Loss of microvascular negative charges accompanied by interstitial edema in septic rats' heart. Circ. Shock 36, 45-56.

Golsch, U., Hager, U., Dorninis, M. and Vestweber, D. (1994) Expression of P-selectin on endothelial cells is up-regulated by LPS and TNF-alpha in vivo. Cell Adhes. Commun. 2, 7-14.

Guan, J.-L. and Hynes, R.O. (1990) Lymphoid cells recognize an alteratively spliced segment of fibronectin via the integrin receptor alpha4-beta1. Cell 60, 53-61.

Hart, M.N., VanDyk, L.F., Moore, S.A., Shasby, D.M. and Cancilla, P.A. (1987) Differential opening of the brain endothelial barrier following neutralization of the endothelial luminal anionic charge in vitro. J. Neuropathol. Exp. Neurol. 46, 141-153.

Hogg, N., Harvey, J., Cabañas, C. and Landis, R.C. (1993) Control of leukocyte integrin activation. Am. Rev. Respir. Dis. 148, S55-S59.

Hughes, C.C.W. and Lantos, P.L. (1986) Brain capillary endothelial cells in vitro lack surface IgG Fc. receptors. Neurosci. Lett. 68, 100-106.

Kavanaugh, A., Lightfoot, E., Lipsky, P.E. and Oppenheimer-Marks N (1991): Role of CD11/CD18 in adhesion and transendothelial migration of $\mathrm{T}$ cells. Analysis utilizing CD18-deficient T cell clones. J. Immunol. 146, 4149-4156.

Klein, N.J., Shennan, G.I., Heyderman, R.S. and Levin, M. (1992) Alteration in glycosaminoglycan metabolism and surface charge on human umbilical cells induced by cytokines, endotoxin and neutrophils. J. Cell Sci. 102, 821-832.

Klein, N.J., Shennan, G.I., Heyderman, R.S. and Levin, M. (1993) Detection of glycosaminoglycan on the surface of human umbilical vein cells using gold conjugated poly-l-lysine with silver enhancement. Histochemical J. 25, 291-298.

Kobayashi, M., Shumada, K. and Ozawa, T. (1990) Human recombinant interleukin $1 \beta$ and tumor necrosis factor $\alpha$ mediated suppression of heparin-like compounds on cultured porcine aortic endothelial cells. J. Cell Physiol. 144, 383-389.

Komoria, A., Green, L.J., Mervic, M., Yamada, S.S., Yamada, K.M. and Humphries, M.J. (1991) The minimal essential sequence for a major cell type-specific adhesion site (CS-1) within the alternatively spliced type III connecting segment domain of fibronectin is leucine-aspartic acid-valine. J. Biol. Chem. 266, 1575-1579.

Lossinsky, A.S., Badmajew, V., Robson, J.A., Moretz, R.C. and Wisniewski, H.M. (1989) Sites of egress of inflammatory cells and horseradish peroxidase transport across the blood-brain barrier in a murine model of chronic relapsing experimental allergic encephalomyelitis. Acta Neuropathol. 78, 359-371.

Lowe, J.B., Stoolman, L.M., Nair, R.P., Larsen, R.D., Berhend, T.L. and Marks, R.M. (1990) ELAM-1-dependent cell adhesion to vascular endothelium determined by a transfected human fucosyltransferase cDNA. Cell 63, 475-484.

Male, D.K., Pryce, G. and Hughes, C.C. (1987) Antigen presentation in brain: MHC induction on brain endothelium and astrocytes compared. Immunology 60, 453-459.

Male, D., Pryce, G., Hughes, C. and Lantos, P. (1990) Lymphocyte migration into brain modelled in vitro: control by lymphocyte activation, cytokines and antigen. Cell. Immunol. 27, 1-11.

Male, D., Rahman, J., Pryce, G., Tamatani, T. and Miyasaka, M. (1994) Lymphocyte migration into the CNS modelled in vitro: roles of LFA-1, ICAM-1 and VLA-4. Immunology 81, 366-372. 
May, M.J., Entwistle, G., Humphries, M.J. and Ager, A. (1993) VCAM-1 is a CS-1 peptide-inhibitable adhesion molecule expressed by lymph node high endothelium. J. Cell Sci. 106, 109-119.

Mayhan, W.G., Faraci, F.M., Siems, J.L. and Heistad, D.D. (1989) Role of the molecular charge in disruption of the blond-brain barrier during hypertension. Circ. Res. 64, 658-664.

McGuire, P.G. and Orkin, R.W. (1987) Isolation of rat aortic endothelial cells by primary explant techniques and their phenotypic modulation by defined substrata. Lab. Invest. 57, 94-105.

Mumby, S.M., Abbott-Brown, D., Raugi, G.J. and Bornstein, P. (1984) Regulation of thrombospondin secretion by cells in culture. J. Cell Physiol. 120, 280-288.

Nag, S. (1984) Cerebral endothelial surface charge in hypertension. Acta Neuropathol. 63, 276-281.

Oppenheimer-Marks, N., Davis, L.S., Bogue, D., Ramberg, J. and Lipsky, P.E. (1991) Differential utilization of ICAM1 and VCAM1 during the adhesion and transendothelial migration of human $T$ lymphocytes. J. Immunol. 147, 2913-2921.

Osborn, L., Vassallo, C., Browning, B.G., Tizard, R., Haskard, D.O., Benjamin, C.D., Dougas, I. and Kirchhausen, T. (1994) Arrangement of domains, and amino acid residues required for binding of vascular cell adhesion molecule-1 to its counter-receptor VLA-4 ( $\alpha 4 \beta 1)$. J. Cell Biol. 124, 601-608.

Pryce, G., Male, D.K. and Sarkar, C. (1991) Control of lymphocyte migration into brain: selective interactions of lymphocyte subpopulations with brain endothelium. Immunology 72, 393-398.

Pryce, G., Santos, W. and Male, D. (1994) An assay for the analysis of lymphocyte migration across cerebral endothelium in vitro. J. Immunol. Methods 167, 55-63.

Raine, C.S. (1994) The Dale E. McFarlin Memorial Lecture: The immunology of the multiple sclerosis lesion. Ann. Neurol. 36, S61-S72.

Raine, C.S., Cannella, B., Dvijvestijn, A.M. and Cross, A.H. (1990) Homing to central nervous system vasculature by antigen-specific lymphocytes. II lymphocyte/endothelial cell adhesion during the initial stages of autoimmune demyelination. Lab. Invest. 63, 476-489.

Santos, W.L.C., Rahman, J., Klein, N. and Male, D.K. (1995) Distribution and analysis of surface charge on brain endothelium in vitro and in situ. Acta Neuropath. 90, 305-311.

Schmidley, J.W. and Wissig, S.L. (1986) Anionic sites on the luminal surface of fenestrated and continuous capillaries of the CNS. Brain Res. 363, 265-271.
Shimizu, Y., Newman, W., Gopal, T.V., Horgan, K.J., Graber, N., Beall, D., van Seventer, G.A. and Shaw, S. (1991) Four molecular pathways of $T$ cell adhesion to endothelial cells: roles of LFA-1, VCAM-1, and ELAM-1 and changes in path ways hierarchy under different activation conditions. J. Cell Biol. 113, 1203-1212.

Springer, T.A. (1994) Traffic signals for lymphocyte recirculation and leukocyte emigration: the multistep paradigm. Cell 76, 301-314.

Tanaka, Y., Adams, D.H., Hubscher, S., Hirano, H., Siebenlist, U. and Shaw, S. (1993a) T-cell adhesion induced by proteoglycan-immobilized cytokine MIP-1 $\beta$. Nature $361,79-82$.

Tanaka, Y., Adams, D.H. and Shaw, S. (1993b) Proteoglycans on endothelial cells present adhesion-inducing cytokines to leukocytes. Immunol. Today 14, 111-115.

Turner, G.D., Morrison, H., Jones, M., Davis, T.M., Looareesuwan, S., Bulry, I.D., Gatter, K.C., Newbold, C.I., Pukritayakamee, S., Nagachinta, B., White, N.J. and Berendt, A.R. (1994) Immunohistochemical study of the pathology of fatal malaria. Evidence for widespread endothelial activation and potential role of intercellular adhesion molecule-1 in cerebral sequestration. Am. J. Pathol. 145, 1057-1069.

Vorbrodt, A.W., Dobrogowska, D.H., Lossinsky, A.S. and Wisniewski, H.M. (1990) Changes in the distribution of anionic sites in brain micro-blood vessels with and without amyloid deposits in scrapie-infected mice. Acta Neuropathol. 79, 355-363.

Vorbrodt, A.W. and Trowbridge, R.S. (1993) Aluminium-induced alteration of surface anionic sites in cultured brain microvascular endothelial cells. Acta Neuropathol. 86, 371-377.

Weller, P.F., Rand, T.H., Goelz, S.E., Chi-Rosso, G. and Lobb, R.R. (1991) Human eosinophyl adherence to vascular endothelium mediated by binding to vascular cell adhesion molecule 1 and endothelial leukocyte adhesion molecule. Proc. Natl. Acad. Sci. USA 88, 74307433

Yabkowitz, R., Dixit, V.M., Guo, N., Roberts, D.D. and Shimizu, Y. (1993) Activated T-cell adhesion to thrombospondin is mediated by the alpha-4 beta-1 (VLA-4) and alpha-5 beta-1 (VLA-5) integrins. J. Immunol. 151, 149-158.

Yednock, T.A., Cannon, C., Fritz, L.C., Sanches-Madrid, F., Steinman, L. and Karin, N. (1992) Prevention of experimental autoimmune encephalomyelitis by antibodies against $\alpha 4 \beta 1$ integrin. Nature 356, $63-66$. 